\section{Modelo de indicadores de valor agregado de plantas medicinales para elevar el potencial exportador}

\author{
Model of value added indicators of medicinal plants to raise \\ export potential
}

\begin{abstract}
RESUMEN
La presente tesis busca incrementar el potencial exportador de las empresas que elaboran productos derivados de las plantas medicinales, mediante el diseño de un modelo obtenido de la relación entre el valor de las exportaciones y el comportamiento de los indicadores de valor agregado. Con información recopilada de fuente primaria y secundaria, se pudo conocer ampliamente el tema y plantear las hipótesis sobre los indicadores y el diseño del modelo. Dichas hipótesis fueron comprobadas mediante el análisis estadístico de los datos extraídos; logrando confirmar que los indicadores planteados eran los correctos y cuantificar en qué medida aporta cada uno de ellos al valor de las exportaciones, para más adelante formular matemáticamente la relación entre el comportamiento de dichos indicadores y el valor de las exportaciones, lográndose un modelo que relacione eficientemente estas dos variables.
\end{abstract}

Palabras claves: Indicadores de Valor; medicina alternativa; oferta exportable; plantas medicinales; valor agregado.

\begin{abstract}
This the sisseeks to increase the export potential of companies that produce medicinal plants products by designing model obtained from the ratio between the export value and the behavior of added value indicators. With information collected from primary and secondary sources, it was possible to know the subject extensively and to propose the hypothesis about the indicators and the design of the model. These hypotheses were verified by the statistical analysis of the extracted data; confirming that the indicators were correct and quantifying, the extent to which each of them contributed to the value of exports, to later formulate mathematically the relationship between the behavior of these indicators and the value of exports, achieving a model that efficiently relates these two variables.

Keywords: Value Indicators; alternative medicine; exportable offer; medicinal plants; value added.
\end{abstract}

\section{César Rafael Sotomayor Sancho Dávila}

csotomayorsd@gmail.com

Universidad Nacional Mayor de San Marcos, Facultad de Ciencias Administrativas

(C) Los autores. Este artículo es publicado por Gestión en el Tercer Milenio de la Facultad de Ciencias Administrativas de la Universidad Nacional Mayor de San Marcos. Este es un artículo de acceso abierto, distribuido bajo los términos de la licencia Creative Commons Atribucion - No Comercia_Compartir Igual 4.0 Internacional. (http://creativecommons.org/licenses/by-nc-sa/4.0/) que permite el uso no comercial, distribución y reproducción en cualquier medio, siempre que la obra original sea debidamente citada. 


\section{INTRODUCCIÓN}

El Perú ha efectuado importantes aportes de especies y variedades para el mundo puesto que cuenta con una gran diversidad de pisos ecológicos y microclimas, donde habría $50 \mathrm{mil}$ especies vegetales, de las cuales 2,000 han sido utilizadas con fines curativos.

Esta riqueza, junto al conocimiento ancestral del uso etnofarmacológico de estos vegetales, constituye un valioso recurso por explotar en beneficio de la humanidad $y$, especialmente, de las comunidades nativas que han preservado estos recursos.

De estas plantas medicinales, se vienen identificando algunos cultivos promisorios, entre los que se encuentran el sacha inchi, maca, yacón, tara y otros. Adicionalmente, algunos de ellos cuentan con certificación orgánica.

A lo largo de esta investigación se pretende desarrollar el diseño de un modelo que permitirá incrementar el potencial exportador de las empresas que elaboran productos derivados de plantas medicinales, al aumentar su nivel de valor agregado en la cadena de valor.

\section{Objetivo general}

La presente investigación pretende proponer un modelo de indicadores de valor agregado de los productos derivados de plantas medicinales para elevar el valor de sus exportaciones.

\section{Objetivos específicos}

- Determinar la relación entre el valor de las exportaciones de los productos derivados de plantas medicinales y su valor agregado.

- Determinar cuáles son los indicadores que condicionan el valor agregado de las empresas que exportan productos derivados de plantas medicinales.

- Determinar la relación entre el valor de las exportaciones de los productos derivados de plantas medicinales y cada uno de los indicadores de valor agregado.

\section{PANORAMA DEL ESTUDIO}

La investigación permite analizar y conocer indicadores de valor agregado de los productos de exportación agrícolas de origen en las Plantas Medicinales, con el propósito de aprovechar nuestras plantas medicinales peruanas; asimismo, el posterior desarrollo de un modelo basado en dichos indicadores favorecerá a un grupo de empresas que corresponden al sector exportador farmacéutico y agropecuario, aproximadamente 50 y 120 empresas respectivamente (SIICEX, 2011).

$\mathrm{Si}$ bien es cierto este número es menor comparado con otros sectores, esto se debe al escaso desarrollo de la industria farmacéutica que no va acorde a la diversidad de plantas con propiedades curativas que crecen dentro del territorio de nuestro país y que, junto al conocimiento ancestral que se ha acumulado durante siglos sobre estas plantas, crea un potencial inmenso que está siendo poco aprovechado por las empresas peruanas, en contraste con las empresas extranjeras que detectan oportunidades aprovechándolas libremente.

La presente investigación busca elevar la oferta de este sector de empresas incrementando el valor agregado en sus productos y convirtiendo su oferta exportable más atractiva para el creciente mercado internacional; lo que tendrá incidencia en las exportaciones de productos a base de plantas medicinales y por ende en las exportaciones del país. Además, contribuye en el desarrollo de la industria farmacéutica peruana, fortaleciendo a las empresas actuales además de motivar la generación de nuevas unidades económicas en dicho sector.

\section{REFERENCIAS TEÓRICAS Y DE ANTECEDENTES POR VARIABLES}

\section{Cadena de valor agregado}

Para las empresas, el valor está compuesto por el ingreso que se puede lograr, menos los costos de generar ese ingreso. En el caso de los clientes, el valor está en los beneficios que le aportamos, menos los costos por obtener esos beneficios. Entre estas dos fórmulas, está el "valor económico creado" (Bindi, 2009, p.194). 
Paredes, M., Hernández, H., Vicuña, L. y Rivera, J. (2009) definen al valor agregado o valor añadido como una característica o servicio extra que se le da a un producto o servicio, con el fin de darle un mayor valor comercial; generalmente se trata de una característica o servicio poco común o poco usado por los competidores y que le da al negocio o empresa cierta diferenciación, comparado con los competidores y entre las diferentes etapas de producción.

El análisis de la cadena de valor agregado es un método utilizado para descomponer la cadena en cada una de las actividades que la conforman, desde la recepción de la materia prima hasta el cliente; con la finalidad de entender el comportamiento de los costos y las fuentes de diferenciación del producto, y para tratar de maximizar la diferencia entre compras y ventas, con el objeto de crear el mayor valor agregado que permita maximizar las utilidades y así ser más fuerte en el mercado.

Castellanos, Ramírez, Fúquene, Quintero y Fonseca (2013), indican que:

Innovar no solo significa desarrollar nuevos productos y transformar los productos existentes; consiste en crear nuevas formas de organizar, gestionar, producir, entregar, comercializar, vender y relacionarse con clientes y proveedores; logrando generar valor agregado a través de toda la cadena productiva. (p.197).

\section{Análisis del valor}

El análisis de valor se puede definir como un proceso de revisión sistemática, que se aplica a los diseños de los productos existentes para compararlos con las funciones de los productos que solicitan los clientes y así satisfacer sus exigencias al menor coste, ofreciendo un rendimiento concreto y la fiabilidad exigida. Tal como lo comentan Escorsa y Valls (2003, Cap. 8) en su libro "Tecnología e Innovación en la Empresa" resumidamente este método busca reinventar el producto para que cumpla específicamente las necesidades del cliente, al mínimo costo posible.

Ampliando esta definición, el análisis de valor es un complejo método para mejorar el valor de un artículo (entendido como relación entre una función destinada a satisfacer al cliente y el coste de dicha función) o proceso, entendiendo los elementos que lo constituyen y sus costes asociados, y tratando después de mejorar los componentes, bien reduciendo su coste o bien incrementando el valor de las funciones.

\section{Tratamiento del valor agregado por Aduanas}

Según la última Ley General de Aduanas Decreto Legislativo $\mathrm{N}^{\circ} 1053$ y su respectivo reglamento (2009), el valor agregado es aquel aumento de valor comercial generado por la transformación de la materia prima e insumos, generalmente en un proceso de maquila.

El valor agregado es un concepto que tiene un tratamiento definido en los procedimientos aduaneros, específicamente para el desarrollo de cuatro de los catorce regímenes aduaneros existentes actualmente en el Perú, los que están referidos a:

- Admisión temporal para el perfeccionamiento activo

- Exportación temporal para el perfeccionamiento pasivo

- Reposición de franquicias arancelarias

\section{- Drawback}

De esto infiere que el tratamiento del valor agregado por la autoridad aduanera tiene el objetivo de determinar correctamente cuál es el valor adicional generado por las actividades de producción, transformación, ensamblaje o maquila realizada dentro del territorio nacional o fuera de él. Para ello aplica la lógica de restar el valor comercial del producto compensatorio o producto final, con el valor comercial de la materia prima e insumos nacionales adquiridos para obtener el producto final.

Es en base a esta lógica que se planteará, más adelante, el modelo de valor agregado que da motivo a esta investigación. Ahondando más en el cálculo monetario de este valor agregado, se procede a exponer el principal mecanismo empleado por los operadores del comercio exterior relacionados a cualquiera de los cuatro regímenes mencionados anteriormente, para sustentar formalmente ante la autoridad aduanera; el valor monetario correspondiente al valor agregado en sus productos. A este se le denomina Cuadro de coeficiente insumo producto. 
Toda esta información contenida en el cuadro de coeficiente insumo producto, tiene carácter de declaración jurada y en base a ella la autoridad aduanera determina los tributos por cobrar o restitución al importador de los insumos o materias primas generadoras de productos para la exportación, siempre y cuando el ministerio competente haya, determinado como válida, la información declarada.

\section{METODO}

\subsection{Tipo}

El estudio de investigación se orienta hacia el tipo de investigación "no experimental", pues no se manipulan las variables intencionalmente, sino se observan los fenómenos tal y como se dan en su contexto natural.

Entre los diferentes tipos de investigación no experimental se ha seleccionado el "correlacional", Pues a investigación trata de relacionar la utilización de indicadores de valor agregado en las empresas elaboradoras de productos basados en plantas medicinales, con su incidencia sobre las exportaciones anuales.

\subsection{Diseño de investigación}

Para guiar el diseño de la investigación se elaboró una matriz de consistencia, mostrada en la Tabla $\mathrm{N}^{\circ} 1$, donde se relacionan objetivos e hipótesis y se determinan variables e indicadores que más adelante formarán parte del modelo de gestión empresarial que planea esta investigación.

\section{RESULTADOS}

Como se mencionó en acápites anteriores, la demostración de las hipótesis específicas tiene la consecuencia automática de la demostración de la conjetura general o principal. Por ello, se han planteado las hipótesis específicas necesarias para cumplir con lo mencionado.

Tabla 1: Matriz de Consistencia de la Investigación

\begin{tabular}{|c|c|c|c|c|c|c|}
\hline $\begin{array}{c}\text { FORMULACIÓN } \\
\text { DEL } \\
\text { PROBLEMA }\end{array}$ & OBJETIVOS & HIPÓTESIS & VARIABLES & INDICADORES & $\begin{array}{l}\text { TÉCNICA DE } \\
\text { RECOPILACIÓN } \\
\text { DE DATOS }\end{array}$ & $\begin{array}{c}\text { FUENTE / } \\
\text { INFORMANTES }\end{array}$ \\
\hline \multirow{2}{*}{$\begin{array}{l}\text { Problema } \\
\text { General: } \\
\text { ¿En qué medida } \\
\text { el incremento del } \\
\text { valor agregado, } \\
\text { en base a } \\
\text { indicadores, de } \\
\text { los productos } \\
\text { derivados } \\
\text { de plantas } \\
\text { medicinales } \\
\text { influirá en el } \\
\text { valor de sus } \\
\text { exportaciones? }\end{array}$} & \multirow{2}{*}{$\begin{array}{l}\text { Objetivo } \\
\text { General: } \\
\text { Proponer un } \\
\text { modelo de } \\
\text { indicadores } \\
\text { de valor } \\
\text { agregado de } \\
\text { los productos } \\
\text { derivados } \\
\text { de plantas } \\
\text { medicinales } \\
\text { para elevar el } \\
\text { valor de sus } \\
\text { exportaciones. }\end{array}$} & \multirow{2}{*}{$\begin{array}{l}\frac{\text { Hipótesis }}{\text { General: }} \\
\text { El valor de las } \\
\text { exportaciones } \\
\text { de los productos } \\
\text { derivados } \\
\text { de plantas } \\
\text { medicinales } \\
\text { se encuentra } \\
\text { en función al } \\
\text { comportamiento } \\
\text { de los } \\
\text { indicadores de } \\
\text { valor agregado. }\end{array}$} & $\begin{array}{l}\text { Variable } \\
\text { Dependiente: } \\
\text { Y: Valor de } \\
\text { exportaciones }\end{array}$ & $\begin{array}{l}\frac{\text { Indicador }}{\text { de Variable }} \\
\text { Dependiente: } \\
\text { Ratio valor FOB } \\
\text { unitario - costo } \\
\text { de materia } \\
\text { prima }\end{array}$ & $\begin{array}{l}\text { a. Análisis de } \\
\text { registros } \\
\text { b. Encuesta }\end{array}$ & $\begin{array}{l}\text { a. SUNAT } \\
\text { b. Empresas } \\
\text { seleccionadas } \\
\text { aleatoriamente } \\
\text { de las listas de } \\
\text { empresas del } \\
\text { rubro en Perú. }\end{array}$ \\
\hline & & & $\begin{array}{l}\frac{\text { Variable }}{\text { Independiente: }} \\
\mathrm{X:} \\
\text { Comportamiento } \\
\text { de los } \\
\text { indicadores de } \\
\text { valor agregado }\end{array}$ & $\begin{array}{l}\frac{\text { Indicador }}{\text { de Variable }} \\
\text { Independiente: } \\
\text { Índice del } \\
\text { comportamiento } \\
\text { de los } \\
\text { indicadores de } \\
\text { valor agregado }\end{array}$ & Encuesta & $\begin{array}{l}\text { Empresas } \\
\text { seleccionadas } \\
\text { aleatoriamente } \\
\text { de las listas de } \\
\text { empresas del } \\
\text { rubro en Perú. }\end{array}$ \\
\hline \multirow{2}{*}{$\begin{array}{l}\frac{\text { Problema }}{\text { Específico 1: }} \\
\text { ¿Cuál es la } \\
\text { relación entre } \\
\text { el valor de las } \\
\text { exportaciones } \\
\text { de los productos } \\
\text { derivados } \\
\text { de plantas } \\
\text { medicinales y } \\
\text { su nivel de valor } \\
\text { agregado? }\end{array}$} & \multirow{2}{*}{$\begin{array}{l}\text { Objetivo } \\
\text { Específico 1: } \\
\text { Determinar la } \\
\text { relación entre } \\
\text { el valor de las } \\
\text { exportaciones } \\
\text { de los } \\
\text { productos } \\
\text { derivados } \\
\text { de plantas } \\
\text { medicinales } \\
\text { y su nivel } \\
\text { de valor } \\
\text { agregado. }\end{array}$} & \multirow{2}{*}{$\begin{array}{l}\text { Hipótesis } \\
\text { Específica 1: } \\
\text { El valor de las } \\
\text { exportaciones } \\
\text { de los productos } \\
\text { derivados } \\
\text { de plantas } \\
\text { medicinales está } \\
\text { directamente } \\
\text { relacionado a } \\
\text { su nivel valor } \\
\text { agregado. }\end{array}$} & $\begin{array}{l}\text { Variable } \\
\text { Dependiente: } \\
\text { Y: Valor de } \\
\text { exportaciones }\end{array}$ & $\begin{array}{l}\frac{\text { Indicador }}{\text { de Variable }} \\
\text { Dependiente: } \\
\text { Ratio valor FOB } \\
\text { unitario - costo } \\
\text { de materia } \\
\text { prima }\end{array}$ & $\begin{array}{l}\text { a. Análisis de } \\
\text { registros } \\
\text { b. Encuesta }\end{array}$ & $\begin{array}{l}\text { a. SUNAT } \\
\text { b. Empresas } \\
\text { seleccionadas } \\
\text { aleatoriamente } \\
\text { de las listas de } \\
\text { empresas del } \\
\text { rubro en Perú. }\end{array}$ \\
\hline & & & $\begin{array}{l}\text { Variable } \\
\frac{\text { Independiente: }}{\text { Z: Nivel de valor }} \\
\text { agregado }\end{array}$ & $\begin{array}{l}\frac{\text { Indicador }}{\text { de Variable }} \\
\text { Independiente: } \\
\text { Índice de valor } \\
\text { agregado }\end{array}$ & $\begin{array}{l}\text { a. Análisis de } \\
\text { registros } \\
\text { b. Encuesta }\end{array}$ & $\begin{array}{l}\text { a. SUNAT } \\
\text { b. Empresas } \\
\text { seleccionadas } \\
\text { aleatoriamente } \\
\text { de las listas de } \\
\text { empresas del } \\
\text { rubro en Perú. }\end{array}$ \\
\hline
\end{tabular}


Modelo de indicadores de valor agregado de plantas medicinales para elevar el potencial exportador

\begin{tabular}{|c|c|c|c|c|c|c|}
\hline $\begin{array}{c}\text { FORMULACIÓN } \\
\text { DEL } \\
\text { PROBLEMA }\end{array}$ & OBJETIVOS & HIPÓTESIS & VARIABLES & INDICADORES & $\begin{array}{c}\text { TÉCNICA DE } \\
\text { RECOPILACIÓN } \\
\text { DE DATOS }\end{array}$ & $\begin{array}{c}\text { FUENTE / } \\
\text { INFORMANTES }\end{array}$ \\
\hline \multirow{2}{*}{$\begin{array}{l}\text { Problema } \\
\text { Específico 2: } \\
\text { ¿Cuáles son los } \\
\text { indicadores que } \\
\text { condicionan el } \\
\text { valor agregado } \\
\text { de las empresas } \\
\text { que exportan } \\
\text { productos } \\
\text { derivados } \\
\text { de plantas } \\
\text { medicinales? }\end{array}$} & \multirow{2}{*}{$\begin{array}{l}\text { Objetivo } \\
\text { Específico 2: } \\
\text { Determinar } \\
\text { cuáles son los } \\
\text { indicadores } \\
\text { que } \\
\text { condicionan } \\
\text { el valor } \\
\text { agregado de } \\
\text { las empresas } \\
\text { que exportan } \\
\text { productos } \\
\text { derivados } \\
\text { de plantas } \\
\text { medicinales. }\end{array}$} & \multirow{2}{*}{$\begin{array}{l}\text { Hipótesis } \\
\text { Especifica 2: } \\
\text { Los } \\
\text { indicadores } \\
\text { de valor } \\
\text { agregado de } \\
\text { las empresas } \\
\text { que exportan } \\
\text { productos } \\
\text { derivados } \\
\text { de plantas } \\
\text { medicinales } \\
\text { son los niveles } \\
\text { de I+D, } \\
\text { tecnología de } \\
\text { procesos y } \\
\text { marketing. }\end{array}$} & $\begin{array}{l}\text { Variable: } \\
\mathrm{X}: \\
\text { Comportamiento } \\
\text { de los } \\
\text { indicadores de } \\
\text { valor agregado }\end{array}$ & $\begin{array}{l}\text { Indicador de } \\
\text { Variable: } \\
\text { Îndice del } \\
\text { comportamiento } \\
\text { de los } \\
\text { indicadores de } \\
\text { valor agregado }\end{array}$ & \multirow{2}{*}{$\begin{array}{l}\text { a. Encuesta } \\
\text { b. Entrevista de } \\
\text { Profundidad }\end{array}$} & \multirow{2}{*}{$\begin{array}{l}\text { a. Empresas } \\
\text { seleccionadas } \\
\text { aleatoriamente } \\
\text { de las listas de } \\
\text { empresas del } \\
\text { rubro en Perú. } \\
\text { b. Entrevista a } \\
\text { Jefe o Gerente } \\
\text { de una empresa. }\end{array}$} \\
\hline & & & $\begin{array}{l}\text { Dimensiones o } \\
\text { componentes de } \\
\text { la variable: } \\
\text { X1: I+D } \\
\text { X2: Tecnología } \\
\text { de Procesos } \\
\text { X3: Marketing }\end{array}$ & $\begin{array}{l}\text { Indicadores de } \\
\text { dimensiones: } \\
\text { - Nivel de I+D } \\
\text { - Nivel de } \\
\text { Tecnología de } \\
\text { Procesos } \\
\text { - Nivel de } \\
\text { Marketing }\end{array}$ & & \\
\hline \multirow{2}{*}{$\begin{array}{l}\text { Problema } \\
\text { Específico 3: } \\
\text { ¿Cómo están } \\
\text { relacionados } \\
\text { el valor de las } \\
\text { exportaciones } \\
\text { de los productos } \\
\text { derivados } \\
\text { de plantas } \\
\text { medicinales y } \\
\text { cada uno de los } \\
\text { indicadores de } \\
\text { valor agregado? }\end{array}$} & \multirow{2}{*}{$\begin{array}{l}\text { Objetivo } \\
\text { Específico 3: } \\
\text { Determinar la } \\
\text { relación entre } \\
\text { el valor de las } \\
\text { exportaciones } \\
\text { de los } \\
\text { productos } \\
\text { derivados } \\
\text { de plantas } \\
\text { medicinales } \\
\text { y cada } \\
\text { uno de los } \\
\text { indicadores } \\
\text { de valor } \\
\text { agregado. }\end{array}$} & \multirow{2}{*}{$\begin{array}{l}\text { Hipótesis } \\
\text { Específica 3: } \\
\text { El valor de las } \\
\text { exportaciones } \\
\text { de los } \\
\text { productos } \\
\text { derivados } \\
\text { de plantas } \\
\text { medicinales } \\
\text { está en } \\
\text { directamente } \\
\text { relacionado } \\
\text { con cada } \\
\text { uno de los } \\
\text { indicadores } \\
\text { de valor } \\
\text { agregado. }\end{array}$} & $\begin{array}{l}\text { Variable } \\
\text { Dependiente: } \\
\text { Y: Valor de } \\
\text { exportaciones }\end{array}$ & $\begin{array}{l}\frac{\text { Indicador }}{\text { de Variable }} \\
\text { Dependiente: } \\
\text { Ratio valor FOB } \\
\text { unitario - costo de } \\
\text { materia prima }\end{array}$ & $\begin{array}{l}\text { a. Análisis de } \\
\text { registros } \\
\text { b. Encuesta }\end{array}$ & $\begin{array}{l}\text { a. SUNAT } \\
\text { b. Empresas } \\
\text { seleccionadas } \\
\text { aleatoriamente } \\
\text { de las listas de } \\
\text { empresas del } \\
\text { rubro en Perú. }\end{array}$ \\
\hline & & & $\begin{array}{l}\frac{\text { Variables }}{\text { Independientes: }} \\
\text { X1: I+D } \\
\text { X2: Tecnología } \\
\text { de Procesos } \\
\text { X3: Marketing }\end{array}$ & $\begin{array}{l}\frac{\text { Indicadores }}{\text { de Variables }} \\
\text { Independientes: } \\
\text { - Nivel de I+D } \\
\text { - Nivel de } \\
\text { Tecnología de } \\
\text { Procesos } \\
\text { - Nivel de } \\
\text { Marketing }\end{array}$ & $\begin{array}{l}\text { a. Encuesta } \\
\text { b. Entrevista de } \\
\text { Profundidad }\end{array}$ & $\begin{array}{l}\text { a. Empresas } \\
\text { seleccionadas } \\
\text { aleatoriamente } \\
\text { de las listas de } \\
\text { empresas del } \\
\text { rubro en Perú. } \\
\text { b. Entrevista a } \\
\text { Jefe o Gerente } \\
\text { de una empresa. }\end{array}$ \\
\hline
\end{tabular}

Elaboración propia

Cuadro Resumen de las Hipótesis de la investigación

\begin{tabular}{|c|c|c|c|}
\hline HIPÓTESIS & $\begin{array}{l}\text { OBJETIVO DE LA } \\
\text { HIPÓTESIS }\end{array}$ & PROPOSICIÓN & MÉTODO PARA DEMOSTRAR \\
\hline $\begin{array}{l}\text { Hipótesis } \\
\text { general }\end{array}$ & $\begin{array}{l}\text { Relación entre } \\
\text { comportamiento de los } \\
\text { indicadores de valor } \\
\text { agregado }(\mathrm{X}) \text { y valor de } \\
\text { exportaciones }(\mathrm{Y}) \text {. }\end{array}$ & $\begin{array}{l}\text { "El valor de exportaciones de los } \\
\text { productos derivados de plantas } \\
\text { medicinales se encuentra en función al } \\
\text { comportamiento de los indicadores de } \\
\text { valor agregado en su cadena de valor." }\end{array}$ & $\begin{array}{l}\text { Coeficiente de correlación ( } r \text { ) y modelo } \\
\text { de regresión entre el comportamiento } \\
\text { de los indicadores devalor agregado y el } \\
\text { valor de exportaciones. }\end{array}$ \\
\hline $\begin{array}{l}\text { Hipótesis } \\
\text { específica } 1\end{array}$ & $\begin{array}{l}\text { Relación entre el nivel } \\
\text { de valor agregado } \\
\text { (Z) y el valor de las } \\
\text { exportaciones (Y). }\end{array}$ & $\begin{array}{c}\text { "El valor de las exportaciones de } \\
\text { los productos derivados de plantas } \\
\text { medicinales está directamente } \\
\text { relacionado a su nivel de valor } \\
\text { agregado. }\end{array}$ & $\begin{array}{l}\text { Coeficiente de correlación y modelo } \\
\text { de regresión }(r) \text { entre el nivel de valor } \\
\text { agregado y el valor de las exportaciones. }\end{array}$ \\
\hline $\begin{array}{l}\text { Hipótesis } \\
\text { específica } 2\end{array}$ & $\begin{array}{l}\text { Determinación de } \\
\text { indicadores de valor } \\
\text { agregado. }\end{array}$ & $\begin{array}{l}\text { "Los indicadores de valor agregado de } \\
\text { las empresas que exportan productos } \\
\text { derivados de plantas medicinales } \\
\text { son los niveles de I+D, tecnología de } \\
\text { procesos y marketing." }\end{array}$ & $\begin{array}{c}\text { Principio de Pareto ( } 20 \% \text { de los } \\
\text { indicadores de valor agregado generan } \\
\text { el } 80 \% \text { del valor agregado, según los } \\
\text { expertos). } \\
\text { Método Delphi Conclusiones basadas en } \\
\text { opiniones de expertos. }\end{array}$ \\
\hline $\begin{array}{l}\text { Hipótesis } \\
\text { específica } 3\end{array}$ & $\begin{array}{l}\text { Corroboración de la } \\
\text { integridad de modelo } \\
\text { (relación entre cada } \\
\text { indicador y el valor de } \\
\text { las exportaciones) }\end{array}$ & $\begin{array}{l}\text { "El valor de las exportaciones de } \\
\text { los productos derivados de plantas } \\
\text { medicinales está directamente } \\
\text { relacionado con cada uno de los } \\
\text { indicadores de valor agregado." }\end{array}$ & $\begin{array}{c}\text { Coeficiente de correlación y modelo } \\
\text { de regresión (r) entre cada indicador y el } \\
\text { valor de las exportaciones. }\end{array}$ \\
\hline
\end{tabular}

Elaboración propia 
Se procede a validar cada una de las hipótesis específicas, empezando con aquella que propone la existencia de relación directa entre el valor de las exportaciones (Y) y la variable nivel de valor agregado (Z).

4.1.1 Relación entre valor de exportaciones (Y) y nivel de valor agregado (Z)

\section{- Hipótesis específica 1:}

$$
\begin{aligned}
& \mathbf{H}_{0}: \rho_{y z .}=0 \text { (Noexistecorrelación) } \\
& \mathbf{H}_{1}: \rho_{y z .} \neq 0(\text { Existecorrelación })
\end{aligned}
$$

Para determinar el nivel de valor agregado de los productos, variable $\mathrm{Z}$, se identificó los 3 principales productos de cada empresa seleccionada y se los clasificó. Luego, mediante la fórmula explicada en la Gráfica $\mathrm{N}^{\circ} 1$ se determina el nivel de valor agregado promedio de cada empresa.

\section{Nivel de valor agregado de los productos}

Se identificó hasta treintaidós niveles diferenciados por su valor agregado, diseñado desde la presentación fresca a granel hasta la tableta gelatinizado, envasado a granel para el consumidor final.

$$
N V A=\left(\sum_{i=1}^{n} N V A_{i}\right) \div n
$$

Gráfica 1. Fórmula para determinar promedio de nivel de valor agregado

Elaboración propia

Luego de obtener los valores para cada una de las empresas integrantes de la muestra se confrontan con el valor de exportaciones que han obtenido por mantener dicho nivel de valor agregado.

Para determinar el valor de las exportaciones (variable Y) se utilizó el ratio valor FOB unitario - costo de materia prima. Con este fin, se solicitó a cada empresa indicar para cada uno de sus 3 principales productos el porcentaje que representa el costo de la materia prima respecto al valor FOB unitario. Para obtener el valor de las exportaciones, se utiliza la relación inversa; es decir el valor FOB unitario sobre el costo de la materia prima. Luego, mediante la fórmula explicada en la Gráfica $\mathrm{N}^{\circ} 2$ se determina el valor de las exportaciones promedio de cada empresa.

$$
V E X=\left(\sum_{i=1}^{n} V E X_{i}\right) \div n
$$

Gráfica 2. Fórmula para determinar promedio de valor de exportaciones

Elaboración propia

Obtenidos los datos de cada empresa, se procede a identificar el modelo representado por la gráfica de dispersión e identificar el modelo que pueda representar esa relación; así como la determinación del coeficiente de correlación entre el nivel de valor agregado y el valor de las exportaciones, pudiéndose determinar que el valor de "r" es de 0.98. Es decir marcadamente a +1 ; con el propósito de identificar la significancia entre estas dos variables, procedemos a realizar la prueba $t$ para el coeficiente de correlación; siendo el Vp ( $p$ valué) es menor que el nivel de significancia del 5\%, por lo que se rechaza la hipótesis nula y se acepta que existe asociación entre las variables.

\section{DISCUSIÓN}

Con el modelo ya planteado, se profundizan ciertos aspectos de la tesis que podrían dar cabida a discusión sobre la efectividad del modelo o la aplicación del mismo. Entre estos puntos a tratar se encuentra la composición de la variable $\mathrm{X}$ del modelo, denominada "valor de exportaciones". Así también se describen detalladamente los indicadores de valor agregado y los tres niveles de profundidad que comprenden:

\subsubsection{Descripción de la Variable Y}

El valor que puede tomar la variable $\mathrm{Y}$ indica el promedio de los ratios valor FOB unitario -costo de materia prima de los 3 principales productos de la empresa-. Es decir cuánto representa el valor FOB de un producto respecto al costo de la materia prima utilizada, en número de veces. Así un ratio de 2 indicaría que el valor FOB representa dos veces el costo de la materia prima. 
5.1.2 Descripción de niveles de cada Indicadores de X

Se formuló la tabla de calificación de indicadores donde cada indicador presenta 3 niveles, cada uno de estos hace referencia a un nivel de inversión distinto, específicamente cada vez mayor. A continuación, se explican las características de cada uno de los niveles, lo que ayudará a calificar correctamente los indicadores de las empresas y definir el nivel que realmente le corresponde.

\section{- Indicador 1: Nivel de Inversión en I+D}

Este primer indicador tiene el objetivo de determinar qué tantos recursos económicos destina una empresa a la investigación y desarrollo para crear productos o innovar los que ya tienen con el fin de conseguir mayor impacto positivo en la salud de las personas.

- No Inversión en I+D: Se incluye a las empresas que no destinan parte de sus recursos a la innovación y perfeccionamiento de productos, sino que prefiere producir lo que actualmente existe en el mercado.

- Inversiones Esporádicas: Se encuentran contenidas las empresas que realizan pocas investigaciones o de menor envergadura, ya que demanda un bajo presupuesto.

- Investigaciones Frecuentes: Las empresas que destinan importante porcentaje de sus recursos a estudios y de larga duración, pero que de obtener resultados positivos les permitiría incrementar sus ventas a niveles que es justificable dicha inversión.

- Indicador 2: Nivel de Inversión en Tecnología de procesos de producción

Este segundo indicador pretende conocer el grado de sofisticación que emplean las empresas para transformar la materia prima, las plantas medicinales, y obtener el producto final.

- Procesos Artesanales: Se trata del nivel más bajo, pues la tecnología es artesanal y las empresas que se encuentran incluidas en este grupo son generalmente dedicadas a la recolección de estas plantas y no se cuenta con máquinas ni equipos complejos.

- Modernización por exigencia del mercado: Las empresas incluidas en este nivel cuentan con un grado mayor de sofisticación y maquinización de sus procesos, sin embargo solo realizan estas modificaciones cuando el mercado lo exige.

- Modernización constante: Este tipo de empresas cuenta con un grado superior de modernización de sus procesos productivos, tiene mayor nivel de maquinización de tareas y elevan su nivel de tecnología.

\section{- Indicador 3: Nivel de Inversión en Marketing}

Este último indicador propone que, mientras se conozca mejor las necesidades del mercado consumidor, los productos y estrategias empleadas en el mercado competidor; como del proveedor $\mathrm{y}$ distribuidor, se podrá satisfacer mejor al cliente y generar mayor valor agregado.

- Sin actividades de Marketing: Se incluyen a las empresas que no realizan las actividades básicas del marketing, porque no destinan recursos a dichas actividades.

- Actividades de Marketing por personas no especializadas: Se trata de aquellas empresas que muestran interés por desarrollar el marketing, pues son conscientes de sus beneficios; sin embargo, o cuentan con un plan de marketing.

- Área de Marketing: Se incluye a exportadoras que sí examinan los mercados con que se relacionan (consumidor, competidor, proveedor y distribuidor), dichas empresas cuentan con un área de marketing con personal profesional especializado en el desarrollo de las actividades. 


\section{CONCLUSIONES}

- El valor de las exportaciones de los productos derivados de plantas medicinales está directamente relacionado a su nivel de valor agregado.

La relación directa entre estas dos variables indica que a mayor nivel de valor agregado, mayor será el valor de las exportaciones.

- Los indicadores de valor agregado de las empresas que exportan productos derivados de plantas medicinales son los niveles de I+D, tecnología de procesos y marketing.

- El modelo diseñado y propuesto que recoge la hipótesis de la investigación se define como sigue:

(a) $\mathrm{Y}=1.5238 \mathrm{X}^{1.8756}$

(b) $\mathrm{X}=0.30 \mathrm{~A}+0.29 \mathrm{~B}+0.22 \mathrm{C}$

Dónde:

$\mathrm{Y}=$ valor de las exportaciones

$\mathrm{X}=$ comportamiento de los indicadores de valor agregado

A, $\mathrm{B}, \mathrm{C}=$ valor de los indicadores

\section{REFERENCIAS BIBLIOGRAFICAS}

Castellanos, O., Ramírez, D., Fúquene, A., Quintero, R. y Fonseca, S. (2013). Competitividad:Apropiación y mecanismos para su fortalecimiento. Bogotá: Universidad Nacional de Colombia.

Chevallier, Andrew. (1997). Enciclopedia de Plantas Medicinales. Madrid: Acento Editorial.

Kaplan R. y Norton, D. (2002). Cuadro de Mando integral. Madrid: Angular Ediciones.

Kaplan R. y Norton, D. (2006). Alignment: Using the Balanced Scorecard to Create Corporate Synergies. Boston: Harvard Business School Press.

Martín de Castro, Gregorio. (2008). Reputación Empresarial y Ventaja Competitiva. Madrid: ESIC Editorial.

Porter, M. (2002). Estrategias Competitivas: Técnicas para analizar industrias y competidores. México.

Porter, M. (2009). Ser Competitivo: Edición actualizada y aumentada. Barcelona:Editorial Deusto. 